II.

\title{
Ein Fall von isolirter Lähmung des ganzen dritten Trigeminusastes nebst einigen Bemer- kungen iiber den Verlauf der Geschmacksfasern der Chorda tympani und die Innervation des Geschmackes ïberhaupt.
}

Von Dr. Fr. Ziehl in Lübeck.

Fälle von isolirten Lähmungen des ganzen Trigeminus sind, obschon sie nicht zu den alltäglichen Vorkommnissen zählen, doch nichts besonderes Seltenes in der medicinischen Literatur. Sehr viel spärlicher sind die Mittheilangen solcher Fälle, bei denen es sich um die isolirte Lähmung eines einzelnen Astès desselben mit dem Sitz der Erkrankung an der Schädelbasis handelt. Fälle dagegen, wo die Ursache der Lähmung weiter abwärts im peripherischen Verlauf eines Astes oder eines seiner $Z$ weige zu suchen war, sind wieder häufiger, da hier die Nervenfasern oft secundär an den nicht so seltenen Erkrankungen ihrer Umgebung, besonders der Knochen, die sie durchsetzen, theilnehmen. Dass beim Sitz der Erkrankung an der Schädelbasis die Lähmung gewöhnlich alle drei Aeste betrifft, ist ja leicht begreiflich; denn nur ganz kleine Krankheitsheerde werden hier auf dem engen Raum einen Ast treffen können, gewöhnlich sind sie dazu viel zu gross, und alle drei Aeste werden betheiligt. Diese Trigeminuslähmungen an der Schädelbasis haben von jeher die Aufmerksamkeit der Pathologen in besonders hohem Grade erregt wegen der mancherlei interessanten Symptome, die sie machen (neuroparalytische Ophthalmie, trophische Störungen an der Mundschleimhaut u.s. w.), und der Wichtigkeit, die sie haben für die Entscheidung einiger streitiger Fragen aus dem Gebiete der Physiologie. $\mathrm{Zu}$ den letzteren gehört ganz besonders die Frage nach dem ferneren Verbleib der Geschmacksfasern des Lingualis, die bekanntlich zunächst, wie allgemein 
angenommen, in die Chorda tympani übergehen, während dagegen ihr weiterer Verlauf streitig ist.

Ganz besonders selten scheinen Erkrankungen einzelner Aeste des Quintus zu sein, bei denen sich zugleich eine Geschmackslähmung fand. Erb ${ }^{1}$ ) erklärt als beweisend nur einen Fall von Romberg, auf den wir unten zurückkommen. Seitdem sind nicht viele genau beobachtete. Fälle hinzugekommen. Wir wollen im Folgenden eine derartige Beobachtung mittheilen, bei der es sich allein um eine luähmung des dritten Trigeminusastes, die motorische Portion eingeschlossen, handelte und wo zugleich Geschmackslähmung auf den vorderen zwoi Drittel der Zunge vorhanden war. Sämmtliche übrigen Gehirn- bezw. Rückenmarksnerven waren intact.

\section{Ein Fall von isolirter Lähmung des dritten Trigeminusastes an der Schädelbasis.}

Sch., 36jähriger Eisenbahncontrolassistent, bemerkte im Juli 1888 beim Rasiren eines Morgens plötzlich, dass er am linken Kinn das Messer nicht mehr spürte und am nämlicben Tage Mittags auch, dass die linke Seite im Iunde beim Essen gefühllos war und ihm hier auf der Zunge auch der Geschmack feble. Bei genauerer Nachforschung fand er nun auch noch eine gefühllose. Stelle ror dem Ohr. Schon einige Tage vorher hatte er beim Kauen fester Speisen im linken Kiefergelenk Schmerzen gespürt und diese Seite daher beim Essen möglichst vermieden. Seit derselben Zeit traten öfters reissende Sehmerzen auf, deren Sitz die Backe war. Der Kranke ist verheirathet, Vater eines Kindes. Syphilitische Infection wird geleugnet. Er selbst führt mit grosser Bestimmtheit seine Erkrankung darauf zurüek, dass er in einem engen, sehr heissen Bureau zu arbeiten batte, wo gerade die linke Gesichtsseite oft starkem Zuge ausgesetzt war.

Stat. praes. am 23. August 1888. Totale Anästbesie für sämmtliche Gefühlsqualitäten (Berührung, Ijruck, Kälte, Wärme, Schmerz, faradischen Strom) an der linken Hä]fte des Kinns und der unteren Lippe, an der linken Hälfte der Zunge, am linksseitigen unteren Zahnfleisch und den Zähnen daselbst, am Boden der Mundböhle links und an der inneren Fläche der linken Backe, fünfwarkstückgrosse anästhetische Stelle in der Gegend des linken Kiefergelenkes, Anästhesie des Tragus und dèr vorderen Hälfte des äusseren Gehörganges, Anästhesie eines schmalen Hautstreifens, der vom linken Kiefergelenk zum Mundwinkel zieht. Die Sensibilität im Gebiet des 1. und 2. Trigeminusastes ganz normal. Dagegen sind, wie leicht durch das Gefübl festzustellen ist, der M. temporalis und masseter gelähnt und atror

1) Ziemssen, Handbuch der spec. Path. u. Therap. Bd. XII. 1. S: 231 . 
phisch. Letzteres ist besonders deutlich an der stark eingesunkenen linken Temporalgegend. Nach rechts berüber kann der Unterkiefer nicht bewegt werden, ebenso nicht in toto gerade nach vorn, vielmehr weicbt er hierbei nach links ab (Lähmung der Pterygoidei). Weder durch faradische noch durch galvanische Reizung kann am Masseter oder Temporalis eine Contraction erzielt werden. Trophische oder vasomotorische Störungen sind im Bereich des anästhetischen Gebietes nicht rorhanden.

Auf den vorderen zwei Drittel der linken Zungenhälfte feblt jede Geschmacksempfindung (bitter, sauer, salzig, süss, elektrischer Strom), rechts dagegen ist sie bier wie am ganzen Zungengrund erhalten. Dagegen fehlt am Zungengrund der linken Seite die Sensibilität (nur Berübrung, Wärme und Kälte gepräft).

Im linken Ohr ist zuweilen ein Geräusch vorbanden "wie wenn in der Ferne eine Fabrik arbeitet". Die Gehörsschärfe ist dieselbe wie rechts. Der: Ohrspiegel zeigt nichts Abnormes. Auch ist der Kranke niemals ohrenleidend gewesen.

Die såmmtlichen âbrigen Gehirnnerven sind intact. Nur fällt am linken Auge die sebr erweiterte, aber regelmässige Pupille auf, die auf Licht weder direct noch vom anderen Auge aus reagirt. Diese érweiterte Pupille wurde hei Pat. schon vor 8 Jahren zufällig constatirt. Seitdem ist sie unverändert sa geblieben. Eine Ursache dafür weiss er nicht. Sehschärfe rechts wie links, mässige Myopie. Der Kranke leidet oft an Flimmerscotom, im Uebrigen ist er ganz gesund.

Der Kranke trat zunächst einen ihm bewilligten längeren Urlaub an. Anfang December 1888 war der Zustand unverändert derselbe, es war bis dahin Jodkali gebraucht worden. Von jetzt ab wurde der Pat. sechs Mal in der Woche quer durch den Kopf galvanisirt und ausserdem die anästhetisehen Stellen mit dem faradischen Pinsel bebandelt.

26. December. Pat. empfindet subjectiv Stechen in der anästhetiscben Stelle am Kinn. Der Masseter seheint sehwach zu fungiren.

28. December. Masseter fungirt dentlich, M. temporalis schwach.

2. Januar 1889. Der Kranke vermag mit der linken Seite wieder zu beissen.

8. Januar. Beim Faradisiren leichtes Stechen am Kinn. Subjective schmerzende Empfindungen längs des ganzen linken Unterkiefers.

16. Januar. Leichtes stechendes Gefühl beim Faradisiren an sämmtlichen früher anästhetisehen Stellen, auch an der Zungenspitze und dem vordersten Theil des Zungenrandes, der übrige Theil der Zunge noch anästhetisch. Ebenso noch völlige Anästhesie an allen Stellen für Berührung, Wärme und Kälte.

22. Januar. Pat. hatte Gefühl für Kälte beim Waschen am Tragus. Die objective Prüfung ergiebt folgenden interessanten Befund: Temperaturen von $+8-10^{\circ} \mathrm{C}$. werden überall wieder als Kälte empfunden, für Wärme besteht noch völlige Anästhesie (geprüft mit Temperaturen zwischen $30-50^{\circ} \mathrm{C}$.), nur am Kinn wird an jener Stelle Wärme empfunden, wo auch zuerst am 
8. Januar der faradische Strom empfunden. wurde. Das Gefübl für leichte Beräbrung ist hier gleichfalls, aber sehr unsicher vorhanden, besser an den früher anästhetischen Stellen am $\mathrm{Ohr}$, an den übrigen Stellen feblt es, ebenso fehlt noch überall die Schmerzempfindung. An der Zunge wird Kälte empfunden, Wärme und Berührung nicht, der faradische Strom erregt leichtes Stechen, der galvanische Kälteempfindung (trotz angewärmter Elektrode). Geschmaksempfindungen: fehlen noch ganz ${ }^{1}$ ).

30. Januar. An allen Stellen werden sämmtliche Gefüblsqqualitäten wieder unterschieden.

Von nun an war der Fortschritt ein regelmässiger. Ende Februar war der Befund wieder ein ganz normaler, nur die Geschmacksempfindung war noch sehr mangelhaft. Nur sauer und der galvanische Strom (kein Unterschied zwischen Anode und Kathode) wurden an der Zungenspitze geschmeckt und an einer umsebriebenen Stelle des vorderen Zungenrandes salzig und sauer, während im Uebrigen die Geschmackslähmung fortbestand. Der Kranke trat ausser Behandlung.

Unser Patient litt also an einer isolirten Lähmung des ganzen dritten Trigeminusastes, die Lähmung der motorischen Portion war nachweisbar durch die. Lähmung der sämmtlichen Kaumuskeln, die der sensiblen Portion durch die charakteristische Verbreitung der Anästhesie im Gebiete sämmtlicher Zweige des 3. Astes. Hieraus ergiebt sich - auch ohne Bestätigung durch die Section - mit Nothwendigkeit und vollkommen sicher über den Sitz der Erkrankung Folgendes: Im Ganglion Gasseri und weiter centralwärts kann sie ihren Sitz nicht haben; denn es wäre ganz unerklärlich, wie hier von den sensiblen Fasern nur die sämmtlich und total gelähmt sein sollten, die später in den 3 . Ast übergehen, während alle anderen gesund bleiben. Ebenso kann die Erkrankung nicht peripherisch von jener Stelle sitzen, wo der 3. Ast fast mit einem Mal in seine Zweige auseinander geht; denn es wäre ebenso

1) Es bestand also an diesem Tage in exquisiter Weise eine sog. partielle Empfindungslähmung. Die Rückkebr der Empfindung fand offenbar in folgender Reihenfolge statt: zuerst für den faradischen Strom, dann für Kälte, hierauf für Berührung und Wärme, schliesslich für Schmerz. Die Empfindung für Druck wurde nicht genauer geprüft, weil mir deren Prüfung im Gesicht mit zu vielen Fehlerquellen behaftet zu sein schien. Dass bei fehlender Emptindung für warm Kältereize empfunden werden, ist schon öfters beobachtet worden. Genaueres darüber findet sich in Verfassers Aufsatz: "Ueber partielle Empfindungslähmung". Deutsche medic. Wochenscbr. 1889. No. 17. 
seltsam, wenn irgend eine Affection gerade zu gleicher Zeit weiter peripherisch davon alle $Z$ weige desselben ergriffen hätte, ohne einen einzigen frei zu lassen. Das wäre wohl denkbar, wenn Zeichen dafür beständen, dass in der Flügelgaumengrube etwa eine Geschwulst vorhanden wäre. Solche aber fehlen durchaus, auch hätte eine Geschwulst wohl nur allmählich und nach und nach die $Z$ weige durch Druck gelähmt. Hier aber wurde die Anästhesie im ganzen Gebiet des 3. Astes fast zu gleicher Zeit constatirt. Der Sitz der Erkrankung kann daher nur auf der kurzen Strecke gesucht werden, wo die sämmtlichen Fasern zu einem Stamm verschmolzen sind. Dies ist nur der Fall vom Ursprung des 3. Astes aus dem Ganglion Gasseri bis knapp nach seinem Durchtritt durch das Foramen ovale; denn hier zweigen schon die motorischen Zweige ab. Wir kommen später noch genauer auf die Diagnose des Sitzes der Erkrankung zurück.

Ist hierüber kein Zweifel möglich, so bleibt doch die Natur der Krankheit selbst dunkel. Am wahrscheinlichsten ist wobl eine Periostitis in der Umgebung des Foramen ovale oder eine exsudative Entzündung und Schwellung des Neurilemms des Nerven selbst, die während des Verlaufes desselben durch jenen engen Kanal hier zu einer Compression des Nervens führte. Wir denken dabei an die Analogie mit der Facialislähmung, und es wäre auch die Ansicht des Kranken über den Ursprung seines Leidens damit am besten verträglich. Der Verlauf der Lühmung kann wohl als Stütze dieser Ansicht betrachtet werden.

2. Ueber den Verlauf der Geschmacksfasern der Chorda tympani und die Innervation des Geschmackes.

Durch zahlreiche Erfahrungen, besonders schlagend nach Resectionen des Lingualis am Menschen wegen Neuralgie, ist festgestellt worden, dass im Bereich der zwei vorderen Drittel der Zunge der zugehörige $N$. lingualis nicht nur Gefühls- soudern auch Geschmacksnerv. ist. Als nicht minder sicher ist es zu betrachten, dass diese Geschmacksfasern ihm durch die Chorda tympani zugeführt werden.

Die meisten Autoren nehmen an, dass die Chorda sämmtliche Geschmacksfasern enthält, nur Schiff ist der Ansicht, dass 
ein Theil dieser Fasern (beim Hund) zunächst in der Bahn des Lingualis verbleibt.

Ob die Chorda auch sensible Fasern der Zunge zuführt, ist zweifelhaft. Einige Beobachtungen scheinen dafür zu sprechen. Sollte dies der Fall sein, so können es beiläufig bemerkt nur Fasern sein, die der Chorda aus dem 3. Ast des $V$ zugeführt worden sind. Sensible Faseru aus einem anderen Nerven, die später für die Zunge bestimmt wären, enthält sie sicher nicht, wie obiger Fall zeigt, wo die Sensibilität der ganzen linken Zungenhälfte für jede Gefühlsqualität erloschen war.

Wo bleiben nun die in die Chorda eingetretenen Geschmacksfasern? Gehen sie mit dem Facialis zum Gehirn oder verlassen sie ihn wieder und in letzterem Fall, zu welchem anderen Nerven treten sie?

Schon unter den Physiologen bestehen hierüber erhebliche Differenzen.

Nach Schiff verlassen die betreffenden Chordafasern den Facialis etwa in der Höhe des Ganglion geniculi und treten wahrscheinlich auf verschiedenen Bahnen (N. petros. superf. maj. et min., Gangl. spheno-palatinum und oticum u. s. w.) wieder in den $\mathrm{V}^{*}$ zurück und zwar in dessen 2. Ast, mit dem sie zum Gehirn verlaufen; denn intracranielle Durchschneidung 'des ganzen $\mathrm{V}$ oder seiner beiden letzten Aeste hebt den Geschmack an der in Frage stehenden Stelle völlig auf. Exstirpation des Ganglion spheno-palatinum oder Durchschneidung seiner Verbindungen mit dem Facialis einer-, dem Quintus andererseits hebt ebenfalls den Geschmack an der genannten Stelle auf. Durchschneidung der Chorda jedoch vermindert ihn nur; ein Theil der Fasern muss also im Lingualis bleiben. Diese verlassen ihn jedoch durch das Gangl. oticum und gehen in inconstanten Bahnen von letzterem gleichfalls zum 2. Ast.

Dem entgegengesetzt ist Lussana der Ansicht, dass die Chordafasern mit dem Facialis zum Gehirn verlaufen und wahrscheinlich in der Port.' intermedia zu fuden seien, dass dagegen der Stamm des $V$ keine Geschmacksfasern enthalte.

Gegenüber diesen von einander abweichenden Angaben hat Erb versucht, durch eine Zusammenstellung von pathologischen Beobachtungen für den Menschen eine Entscheidung in dieser 
Frage herbeizuführen. Das von ihm beigebrachte und kritisch gesichtete Material hier zu wiederholen, liegt bei der leichten Zugänglichkeit des Aufsatzes im Archiv f. Klin. Medicin Bd. XV keine Veranlassung vor. Auf Grund desselben entscheidet Erb sich dahin, es sei mit einer an Gewissheit grenzenden Wahrscheinlichkeit anzunehmen, dass die an der Peripherie im $\mathrm{N}$. lingualis verlaufenden Geschmacksfasern für die vorderen zwei Drittel der Zunge auch in dem Stamm des $V$ bezw. in der sensiblen Wurzel desselben enthalten sind, dagegen vicht im Stamm des Facialis an der Schädelbasis, während sie allerdings zweifellos alle oder zum grössten Theil in dem Felsentheil des Facialis liegen und durch die Chorda der Zunge zugeführt werden.

Erb's Gründe sind folgende: 1) es giebt sichere Fälle von isolirter, vollständiger Anästhesie des V durch Erkrankung seines an der Schädelbasis liegenden Abschnittes mit gleichzeitiger Aufhebung des Geschmackes an der vorderen Zungenhälfte; 2) die entgegenstehenden Fälle, wo bei völliger Anästhesie des $V$ der Geschmack daselbst erhalten geblieben sein soll, sind alle nicht überzeugend; 3) es giebt Fälle von isolirter vollständiger, peripherischer Facialislähmung mit Aufhebung des Geschmacks auf der vorderen Zungenbälfte; 4) existiren Fälle von Lähmung des Facialis an der Schädelbasis oberhalb des Gangl. geniculi ohne Beeinträchtigung des Geschmackes, die gegentheiligen Beobachtungen sind mehr als zweifelhaft.

Es begleiten also die durch die Chorda in den VII übergetretenen Geschmacksfasern letzteren nur eine Strecke weit - von der Eintrittsstelle der Chorda bis etwa zur Höhe des Gangl. geniculi, dann gehen sie in die Bahn des $V$ zurück.

Als Ausnahmefall von untergeordneter Bedeutung lässt $\mathrm{Erb}$ zu, dass die Fasern der Chorda im Facialis centrifugal verlaufen und auf irgend einer anderen Bahn, wahrscheinlich durch den Ram. communicans zum Auriculo temporalis, ans diesem zum Gangl. oticum und von hier zum Gangl. spheno-palatinum oder Gasseri gelangen. Neuere Beobachtungen zu Gunsten dieser Stich'schen Ansicht ${ }^{1}$ ) liegen nicht vor, Diese Bahn kommt also wohl kaum in Betracht.

1) Charité-Annalen. VIII. S. 69. 
Welches sind nun die Bahnen, auf denen die Geschmacksfasern der Chorda in der Regel zum Trigeminus gelangen?

Nach Erb, der zugesteht, dass die dieser Rückkehr dienenden Bahnen noch nicht genau bekannt sind, können es folgende sein: 1) Vom Gangl. geniculi durch den Petros. superf. major und N. Vidianus zum Gangl. spheno-palatinum und damit zum 2. Ast des V (wie auch Schiff annimmt); 2) directe Verbindungsfäden der Chorda mit dem Gangl. oticum u. s. w.; 3) vom Gangl. geniculi durch den Plexus tympanicus zum Petros. superf. minor, zum Gangl. oticum u. s. w.

Es seien wohl noch mehrere Verbindungswege vorhanden, ebenso für die etwa im Lingualis verbleibenden Geschmacksfasern, und es sei möglich, dass dieselben bei einzelnen Individues in sehr verschiedener Weise benutzt würden.

Als eine Angesichts der Experimente und pathologischen Beobachtungen am V nicht gerade wahrscheinliche Möglichkeit erwähot Erb noch, dass Chordafasern durch das Gangl. oticum und den N. tympanicus zum Glossopharyngeus und mit diesem ins Gehirn gelangen könnten.

Indem Erb zur Erklärung scheinbar paradoxer Fälle noch das Bestehen von Varietäten im Nervenverlauf offen lässt, ist sein Resumé: "Zungengrund, Gaumen und Rachenwandungen werden vom Glossopharyngeus versorgt, die vorderen zwei Drittel der Zunge dagegen vom Lingualis; die Geschmacksfasern dieses Nerven gehen zum grössten (alle?) Theil in die Chorda über, verlaufen dann innerhalb der Facialisbahn bis höchstens zum Gangl. geniculi und treten endlich auf verschiedenen, für den Menschen noch nicht genau bekannten Bahnen wieder zum Trigeminus (2. und 3. Ast) über und verlaufen in dem Stamm dieses Nerven zum Gehirn. Ueber die intracerebralen Bahnen desselben sowie über den eigentlichen centralen Heerd der Geschmacksempfindung ist nichts Genaueres bekannt." Erb entscheidet sich also im Ganzen für die Annahme von Schiff.

Seit dem Erscheinen der Erb'schen Arbeit im Jahre 1875 sind eine ganze Anzahl von Arbeiten über unser Thema hinzugekommen. Vor allem sind es Beobachtungen, die bestätigen, dass für den Menschen die Geschmacksfasern des Lingualis aus der Chorda tympani stammen: Besonders beweisend siud solche 
Fälle, wo durch eine Zerstörung der Chorda in Folge krankhafter Prozesse in der Paukenhöhle oder zufälligen Durchschneidens derselben bei Operationen ein Verlust des Geschmackes (zuweilen auch des Gefühls) auf dem vorderen Abschnitt der Zunge herbeigeführt wurde. Mehr oder weniger gehören hierher u. a. die Beobachtungen von Urbantschitsch ${ }^{1}$ ), $W_{o l f}{ }^{2}$ ), Blau ${ }^{3}$ ) und Schulte ). Somit kanu diese Frage als endgültig erledigt betrachtet werden.

Der Versuch von $\mathrm{Carl}^{5}$ ) auf Grund einer Beobachtung an sich selbst, der Chorda nur einen ganz geringen Antheil an der Geschmacksempfindung zuzuschreiben, ist als misslungen zu betrachten. In seiner Beweisführung spielt die Hypothese keine geringe Rolle, so dass alle nachfolgenden Autoren [z. B. Salomonsohn ${ }^{6}$ ), Schulte] die Beweiskraft des Falles mit Recht leugnen.

Dagegen sind die Ansichten der Forscher äber den weiteren Verbleib der Geschmacksfasern der Chorda nach wie vor sehr getheilt und nach der nenesten Arbeit von Bruns scheint es fast, als seien unsere Kenntnisse darüber mehr als je unsicher. Eulenburg ${ }^{7}$ ) scheint u. a. die (von Erb als unwahrscheinlich bezeichnete) Möglichkeit der Berücksichtigung werth zu sein, dass diese Fasern vom Gangl. geniculi durch den Petros. superfic. minor zum Plexus tympanicus gelangen und von hier durch den $N$. Jacobsoni in die centrale Bahn des Glossopharyngens eintreten.

Auch Lando is ${ }^{8}$ ) hält es für das Wahrscheinlichste, dass die Chordafasern vom IX herrühren. Als Bahnen dafür könne man vermuthen zuerst eine jenseits des For. stylomast.: nehmlich durch den R. communicans cum N. glossoph., der von diesem in einen Ast des Facialis geht. Letztere Vereinigung erkläre

1) Archiv für Ohrenheilkunde. Bd. XIX. S. 135.

2) Zeitschrift für Ohrenheilkunde. Bd. IX. S. 152.

3) Berl. klin. Wochenschr. 1879. No. 45.

4) Zeitschrift für Ohrenbeilkunde. Bd. XV. S. 67.

5) Archiv für Ohrenheilkunde. Bd.X. S. 157.

6) Salomonsohn, Ueber den Weg der "Geschmacksfasern" zum Gehirn. Berl. Dissert. 1888. S. 10 .

7) Lehrbuch der functionellen Nervenkrankbeiten.

8) Lehrbuch der Physiologie. 1880. S. 678. 
die constante Geschmacksstörung nach Verletzung des Facialis am For. stylomast. Diese von Landois citirte Angabe Stich's ist jedoch schon von Erb widerlegt worden, dessen Aufsatz aber Landois nicht zu kennen scheint. Als zweite Verbindung ist die oben auch von Eulenburg erwähnte beschrieben. Als dritte Möglichkeit wird ein Fädchen erwähnt, das vom Gangl. petrosum des IX direct zum Facialisstamm im Fallopischen Kanal führe.

Auch Hermana denkt, weil Trigeminuslähmungen ohne Geschmacksstörungen vorkommen, an einen Uebertritt von Glossopharyngeusfasern durch die Jacobson'sche Anastomose, den Petros. superf. minor and das Gangl. oticum in den Trigeminus.

Dass jedoch die angeblichen Fälle von Trigeminuslähmung an der Schädelbasis ohne Geschmacksstörung einer ernsten Kritik nicht Stand halten, hat Erb nachgewiesen, so dass diese Frage als erledigt betrachtet werden sollte. Trotz alledem werden $z$ wei von diesen Fällen (die Lussana als entscheidend betrachtete) auch von Schulte noch wieder citirt ohne jede Rücksicht auf die Kritik Erb's.

Inzwischen ist die Frage, ob die Chordafasern aus dem Glossopharyngeus stammen könnten, als im negativen Sinn erledigt zu betrachten (ganz abgesehen von den Befunden bei Lähmungen des V), da von Lehmann') ein Fall publicirt ist, bei dem in Folge einer Schädelbasisfractur rechtsseitige Lähmung des Glossopharyngeus entstand, während der Quintus intact war. Im Bereiche des letzteren war die Geschmacksempfindung erhalten, in dem des Glossopharyngeus erloschen; denn am Rande, in der Mitte und in der Gegend der Pap. circumvallatae fehlte rechts der Geschmack vollkommen. Die Sensibilität war auf's Feinste erhalten. Zucker und Chimin wurden gar nicht empfunden, auch nicht in den stärksten Concentrationen, nebenbei ein Beweis, dass diese Substanzen keine Tasteindrücke erregen, sondern allein nur den Geschmack. Säuren und Salze bewirkten auf beiden Seiten Brennen und auf der schmeckenden Seite ausserdem den charakteristischen Geschmack. Ein kleiner Theil der Zungenspitze zeigte rechts Reaction gegen alle Geschmacks-

1) Pflüger's Archiv für Physiologie. XXX. S. 194, eit. nach VirchowUirsch, Jahresbericht 1884. I. S. 195. 
eindrücke, etwa so stark wie Seite und Grund der gesunden Hälfte, etwas schwächer als die entsprechende Seite links.

Es waren in diesem Falle offenbar nicht die vorderen zwei Drittel der Zunge vam Lingualis mit Geschmacksfasern versehen, sondern eine etwas kleinere Partie. Dass dies individuell wechselt, geht auch aus anderen Beobachtungen hervor (z. B. war in Wolf's Fall nur das vorderste Drittel mit Chordafasern versorgt). Ueberhaupt ist die Zungenspitze nicht bei allen Menschen in gleicher Weise geschmacksfähig, wie von Vintschgau ${ }^{1}$ ) nachgewiesen ist. Bei ihm selbst war dies nur in sehr beschränktem Maasse der Fall. Leicht und sicher empfand er nur sauer, weniger gut süss, schlecht salzig, fast gar nicht bitter. Elektrische Reizung der Zungenspitze ergab bei Vintschgau bei keiner Stromesrichtung irgend eine Geschmacksempfindung. Der Strom circulirte entweder durch die Zungenspitze allein oder durch den Körper and die Zungenspitze. Nur Gefühlsempfindungen traten auf. Dagegen hatte Dr. Hoenigschmied, dessen Zunge geschmacksfähig war, auch galvanische Geschmacksempfindungen. Sauer und salzig erregten bei hoher Concentration ausserdem die Gefühlsnerven, süss und bitter dagegen nicht.

Man wird gut thun, bei Geschmacksprüfungen hierauf Rücksicht zu nehmen und daher einerseits bei der Prüfung auf sauer und salzig nicht zu starke Concentrationen anwenden, andererseits bei der elektrischen Prüfung aus einem Mangel der galvanischen Geschmacksempfindung nicht auf einen Mangel der Geschmacksempfindung überhaupt schliessen dürfen.

Der Beobachtung Lehmann's kann ich eine ähnliche hinzufügen, die allerdings, weil der Fall complicirter ist, nicht so schlagend, aber in Bezug auf den uns hier interessirenden Punkt völlig eindeutig ist.

Bei einem 28jäbrigen Mädchen war allmählich eine Lähmung entstanden, die den linksseitigen Abducens, Facialis, Acusticus (letzteren nur wenig), Glossopbaryngeus, Vagus (mit Sicherheit den Laryngeus super. et infer.) und den Hypoglossus umfasste. Im Gebiet des Facialis complete, an der linken Hälfte der Zunge partielle Entartungsreaction. Auf der rechten Seite waren diese Nerren intact, ebenso auf beiden Seiten das 1.-5. Paar. Ganz

1) Pflüger's Arch. Bd. 19 u. 20. Virchow-Hirsch, Jabresbericht 1879. I. S. 188. 
besanders konnte im Bereich des Trigeminus nichts Abnormes nachgewiesen werden. Ueber das zu Grunde liegende Leiden, dessen Anfänge mehrere Jahre zurückdatirten, konnte nichts ermittelt werden. Hier war die Geschmacksempfindung am vorderen Abschnitt der Zunge ganz intact, binten anf der linken Hälfte erloschen, während die Sensibilität hier wenigstens für das Berührungsgefühl gleichfalls intact war. Seit etwa drei Jahren ist in dem Leiden ein Stillstand eingetreten. Die Kranke wurde damals, weil hartnäckiges Erbrechen und Beschwerden beim Schlingen auftraten, mit dem galvanischen Strom behandelt. Beide Symptome verloren sich; ob hierdurch auch der Stillstand des Leidens bewirkt wurde, lasse ich dahingestellt.

Mag es sich dabei nun um eine Kernaffection oder um einen krankhaften Prozess an der Schädelbasis gehandelt haben, das ist für die uns hier allein interessirende Frage völlig gleichgültig; denn in beiden Fällen geht aus der Beobachtung wie aus der von Lehmann mit voller Sicherheit hervor, dass die Geschmacksempfindung am vorderen Zungenabschnitt nicht durch den Glossopharyngeus vermittelt wird. Es müsste denn jemand die Annahme machen wollen, dass der krankhafte Prozess gerade alle Fasern bezw. Ganglienzellgruppen für den vorderen Zungenabschnitt verschont, alle Fasern für den hinteren Abschnitt aber vernichtet hätte.

Wir betrachten daher auch diese Frage als erledigt, trotzdem Carl seinen Fall zu Gunsten dieser Theorie glaubt verwenden $z a$ können. Wir erwähnten den Fall schon oben bei der Frage, ob die Chorda die Geschmacksfasern enthält und mussten ihn für durchans nicht beweisend erklären. Auch in Bezug auf die jetzige Frage ist er es nicht, wie Schulte und Salomonsohn a. a. 0 . ausführlich zeigen. Der Meinung des letzteren schliessen wir uns völlig an. Es ist daher unpöthig, auf den Fall hier noch wieder ausführlich einzugehen ${ }^{1}$ ).

Ebenso wenig wie vom Glossopharyngeus stammen die $\mathrm{Ge-}$ schmacksfasern der Chorda aus dem Facialis, da sie in ihm, wie schon Erb als wahrscheinlich nachgewiesen hat, an der Schädelbasis nicht mehr enthalten sind: Damit übereinstimmen die nach dem Aufsatz von Erb erschienenen Arbeiten Vulpian's ${ }^{2}$ ). Nach Durchschneidung des Facialis sowohl bei seinem Eintritt

1) Eine höchst einfache Deutung dieses Falles, wonach er mit der üblichen Ansicht völlig in Uebereinstimmung ist, folgt später.

2) Virchow-Hirseh, Jahresbericht 1878. I. S. 19. 
in den Meatus aud. intern,, wo die Port. Wrisberg. mitgetroffen wurde, als an seinem wahren Ursprung am Boden der Rautengrube zeigte die nach 20 Tagen untersuchte Chorda schr wenig Veränderungen. Demnach scheint ihm die Chorda weder aus dem Facialis noch aus der Port. intermedia zu entspringen.

Wenn aber die Chordafasern weder aus dem Facialis noch aus dem Glossopharyngeus stammen, so bleibt nur übrig, dass sie später in den Trigeminus zurückkehren. Damit stimmt denn auch die Beobachtung am Menschen überein; denn in allen Fällen von totaler Lähmung des $V$ an der Schädelbasis, die vor der Kritik bestehen können, war, wie Erb gezeigt hat, auch die Geschmacksempfindung an den vorderen zwei Drittel der Zunge aufgehoben.

Dieser Ursprung ist auch nach den Experimenten Vulpian's der wahrscheinlichste: Die Durchschneidung des V im Schädel, sagt er, sei sehr schwer, meist werde der VII mit verletzt. Alsdann war die Chorda mehr oder weniger degenerirt. Bei der Durchschneidung des $\mathrm{V}$ allein waren die Resultate verschieden, wahrscheinlich deswegen, weil ein grösserer oder kleinerer Theil unverletzt blieb. Ein Fall erscheine jedoch beweisend, wo der ganze $\mathrm{V}$ bei unverletztem VII durchschnitten war und die Chorda als völlig degenerirt befunden wurde. Ihm scheint also dieselbe vom $V$ zu entspringen.

Von neueren, für diese Ausicht sprechenden Beobachtungen über Lähmungen des $\mathrm{V}$ an der Schädelbasis mit Geschmacksstörungen erwähne ich nar die Fälle von Senator ${ }^{1}$ ), Heusner ${ }^{2}$ ), Salomonsohn ${ }^{3}$ ), Ruhemann ${ }^{4}$ ), Müller ${ }^{5}$ ), Zenner ${ }^{6}$ ) und einen neueren Fall von $\mathrm{Erb}^{7}$ ) selbst, der völlig beweisend wäre, da die Diagnose durch die Section bestätigt werden konnte, wenn nicht - wie übrigens Erb selbst hervorhebt - das Gehörorgan erkrankt gewesen wäre. Derselbe Einwand trifft Heusner's Beobachtung.

1) Berl. klin. Wochenschr. 1881. No.6. 1883. No. 14.

2) Berl. klin. Wochenschr. 1886. No. 44.

3) a. a. 0.

4) Centralbl. f. klin. Medicin. 1889. No. 1.

5) Archiv für Psychiatrie. XIV. S. 263.

$\left.{ }^{6}\right)$ Neurolog. Centralbl. 1888. No. 16.

7) Neurolog. Centralb!. 1882. No. 4 u. 7. 
Ehe wir uns nun zur Beantwortung der Frage wenden, in welchem der drei Aeste des $V$ die Geschmacksfasern enthalten sind, wobei sich zugleich Gelegenheit finden wird, auf einzelne von diesen Fällen genauer einzugehen, müssen wir uns noch mit einer Angabe beschäftigen, die eine bisher noch nicht ausgesprochene, ganz neue Ansicht über das in Frage stehende Thema vertritt. Nehmlich gelegentlich der Mittheilung seines oben erwähnten Falles von Durchtrennung der Chorda gelangt Schulte "nach eingehender Prüfung der Literatur" zu dem Ergebniss, dass die Chorda weder vom V noch vom VII abstamme, dass sie vielmehr zu betrachten sei als peripherisches Ende eines von Sapolini entdeckten, in den Seitensträngen des 4 . Ventrikels entspringenden 13. Hirnnervens; denn wie schon von Inzani und Lussana angenommen und von Sapolini 1873 anatomisch nachgewiesen sei, habe die Chorda bezw. ihre dem Centrum zustrebende Fortsetzung weder mit dem Facialis noch mit dem sie ebenfalls eine Strecke begleitenden Acusticus etwas gemein als den streckenweise gleichen Weg. Sie sei überall als selbständiger Nerv zu isoliren, auch gehe sie weder mit dem Facialis im Gangl. geniculi noch mit irgend einem anderen Nerven eine Anastomose ein; vielmehr will Sapolini diesen Nerven, von dem sowohl der $\mathrm{N}$. intermedius als auch die Chorda nur peripherische Enden vorstellen, von seinem Ursprung durch das G. geniculi hindurch bis zu den äussersten Verzweigungen der Chorda in der Zunge anatomisch verfolgt haben. Diesem 13. Gehirnnerven schreibt Schulte die Function als Geschmaeksnerv für die vorderen zwei Drittel der Zunge zu.

Mag es sich nun mit diesem 13. Gehirnnerven Sapolini's verhalten, wie es will - der gleichen Meinung wie Sapolini sind Bigelow, Spitzka, Burnett, während Vulpian sich wieder dafür entscheidet, dass die Chorda in aller Form eine Abtheilung des Facialis darstelle und gänzlich unter dem Einfluss des Gangl. geniculi stehe - so ist dennoch die Annahme Schulte's, dieser Nerv sei der Geschmacksnerv, deswegen gänzlich unbegründet, weil, wie die obigen Betrachtungen lehren, seine Angabe falsch ist, dass die Abstammung der Chorda sowohl vom $\mathrm{V}$ als auch vom VII deshalb ausgeschlossen sei, weil Durchschneidungen dieser beiden Nerven an ihrem Ursprung in 
der Schädelhöhle weder Aufhebung der Function der Chorda noch Degeneration ihrer Fasern im Gefolge habe. Gerade umgekehrt fand Vulpian solche degenerirte Fasern allerdings nicht bei Durchschneidung des VII, wohl aber bei Durchschneidung des V.

Wie Schulte bei seiner Annahme die Fälle erklärt, wo bei alleiniger Erkrankung des $V$ an der Schädelbasis der Geschmack auf den vorderen zwei Drittel der Zunge fehlt, erfährt man nirgends. Diese in nicht geringer Zahl ganz sicher constatirten Fälle, die mit seiner Annahme in einem unlösbaren Widerspruch stehen, schweigt Schulte einfach todt. Ihre aber nicht zu leugnende Existenz macht seine Annahme zu einer ganz unhaltbaren.

Es bleibt also als einzig sicheres Resultat unserer bisherigen Betrachtung bestehen, dass die Geschmacksfasern, wie schon Erb gezeigt hat, ursprünglich im $V$ liegen und vom $V$ erst in die Chorda herübertreten.

Es fragt sich nun, in welchem Ast des $\mathrm{V}$ sind sie enthalten? Offenbar kommt der erste nicht in Betracht, es kann sich nur um den 2. oder 3. Ast handeln. Beide Ansichten haben ihre Vertreter gefunden. Die meisten plädiren für den 2. Ast.

Hierfür entschied sich schon auf Grund seiner Experimente, wie oben erwähnt, Schiff. Er lässt die Geschmacksfasern durch den Petros. superf. maj., den N. Vidianus und des Gangl. sphenopalatinum zum 2. Ast gelangen. Dieser Ansicht wurde von Prévost widersprochen. Beim Hunde fand er nach Exstirpation des G. spheno-palatinum keine Veränderung des Geschmackes auf derselben Seite. Schiff und Prévost stehen sich also in ihren Ansichten diametral gegenüber.

$\mathrm{Zu}$ Gunsten des 2. Astes hat sich auch Erb ausgesprochen und ganz derselben Ansicht wie Schiff ist Seeligmüller ${ }^{1}$ ).

Neuerdings fand diese Ansicht für den Menschen einen Vertreter in Heusner ${ }^{2}$ ) auf Grund folgender Beobachtung:

Bei einem 52jährigen Arbeiter traten anfallsweise Schmerzen auf, die anfangs auf den 2. Ast des linken $V$ beschränkt, allmählich auch auf das Gebiet des 1. und schliesslich selbst auf einen Theil des 3 . Astes übergingen (Auriculotempor). Es entwickelte sich linksseitige Amaurose, Thränen-

1) Lehrbuch der Krankheiten der peripheren Nerven. S. 122.

3) a. a. 0 . 
träufeln und Speichelfinss. Bald nach der Aufnabme wurde constatirt: Abnahme des Geruchssinnes, Lähmung der linksseitigen Augenbewegungsnerven, Abnahme der Sensibilität links und des Geschmackes. Schliesslich war der Status folgender: Linker Bulbus unbeweglich, amaurotisch, neuroparalytische Ophthalmie, Geruch links erloschen, vollkommene Anästhesie des 1. und 2. Trigeminusastes. Das Geschmacksvermögen auf der linken Zungenbälfte vollkommen erloschen, dagegen Sensibilität im Gebiet des 3. linken Trigeminusastes im Gesicht wie auf der Zunge intact, ebenso Gefühl und Geschmack auf der binteren Zungenbälfte beiderseits, intact Facialis und $N$. Vidianus (Gaumensegel nicht gelähmt, keine Hyperacusis, keine Verminderung sondern im Gegentheil refectorische Vermehrung der Speichelsecretion), intact Gehör und Hypoglossus. Später noch Schwerbörigkeit, doch blieb das VerhäItniss im Gebiet der Trigeminusäste und des Geschmackes bis zuletzt unverändert. Tod an Phthise, keine Section. Vom 11.-23. Lebensjabr bestand Eiterausfluss aus dem linken Ohr, der dann geheilt war, obne Störungen des Gehörs oder anderer Art zu hinterlassen.

Heusner's Diagnose lautet: tuberculöse Caries der Schädelknochen, die von den vorderen und seitlichen Partien des Keilbeins ihren Ausgang nahm und sich von hier aus ausbreitete, das Foramen ovale mit dem 3. Ast des $V$ aber gar nicht oder nur wenig afficirte, ebenso die weiter hinten gelegenen Knochenund Nervenbezirke intact liess. Einen Zusammenhang mit dem früheren Ohrenleiden leugnet $\mathrm{Heusuer}$, auch die Geschmacksstörung könne nicht etwa von früher her bestanden haben, da dieselbe von dem intelligenten und ängstlich auf die Fortschritte der Krankheit passenden Patienten erst im Verlauf des Krankenhausaufenthaltes entdeckt worden sei.

Da hier also nach Heusner's Ansicht eine Erkrankung vorliegt, die den 1. und 2. Ast des V ergriff, den 3. Ast nebst VII und IX aber freiliess, während das Geschmacksvermögen der vorderen Zungenhälfte erloschen war, so sieht er darin eine völlige Bestätigung der Schiff-Erb'schen Annahme über den Verlauf der Geschmacksfasern.

Trotz der Verwahrung Heusner's bemerkt jedoch schon der Referent im Virchow-Hirsch'schen Jahresbericht (Grünhagen), es sei keine Bürgschaft dafür vorhanden, ob nicht die Geschmacksstörung doch schon vor Eintritt der letzten Erkrankung bestanden habe als Folge der früheren eitrigen Ohrenentzündung. Da nun bei solchen Ohrenerkrankungen Alterationen bezw. Verlust des Geschmackes sehr häufig sind (Urbantschitsch), 
auch vorkommen, ohne dass die Kranken etwas davon wissen (cf. Ruhemann's Fall), so müssen wir uns dieser Beanstandung des Falls durch Grünhagen anschliessen. Beweisend wäre er nur, wenn objectiv constatirt wäre, dass Geschmacksempfindungen, ehe der Prozess am V sich entwickelte, noch vorhanden waren, der Kranke könnte dies doch früher sehr wohl, wo er sich noch nicht so ängstlich beobachtete, übersehen haben. Aber ganz abgesehen hiervon könnte Heusner's Fall auch nur das Vorhandensein der Geschmacksfasern im Trigeminusstamm überhaupt beweisen, nicht dagegen, dass sie im 2. Ast verlaufen; denn in der Krankengeschichte ist ausdrücklich angegeben, dass neuralgische Schmerzen auch im Gebiet des 3. Astes vorhanden waren. Derselbe war also auch erkrankt. Wenn nun auch in seinem Gebiet die Sensibilität intact war, so könnten doch sehr wohl etwaige in ihm verlaufende Geschmacksfasern schon zerstört gewesen sein, da die Widerstandskraft der verschiedenen Nervenfasern unter Umständen sehr verschieden ist, wie die Fälle von partieller Empfindungslähmung und auch unser obiger Fall zeigen, bei dem das Gefühl eher zurückkehrte als die Geschmacksempfindung.

Wenn somit der Heusner'sche Fall in doppelter Beziehung angreifbar ist, so ist es um nichts besser bestellt mit der Beobachtung von Salomonsohn ${ }^{1}$ ) (der übrigens wegen der früheren Ohreneiterung den Heusner'schen Fall gleichfalls als nicht beweiskräftig ansieht). Sie scheint mir allerdings zu beweisen, dass die Geschmacksfasern der Chorda im 'Trigeminus liegen, aber dass sie, wie Salomonsohn will, im 2. Ast liegen, beweist sie nicht. Dieser von Mendel beobachtete Fall ist kurz folgender:

37 jähriger Mann, der nie an Obrenaffectionen gelitten batte, erkrankte mit heftigen rechtsseitigen Kopfschmerzen, nach einigen Monaten nabm das Sebvermögen des rechten Auges bis zur völligen Erblindung ab, seit längerer Zeit Taubheitsgefühl unterhalb des rechten Auges. Objectiver Befund: das rechte Auge etwas vorgetrieben, rechte Pupille etwas weiter als die linke, gegen Licht unempfindlich, Augenhintergrund normal. Rechts fehlt die Geruchsempfindung, auf den vorderen zwei Dritteln der Zunge der Geschmack für saner, süss und salzig ganz. Bei Chinin giebt Pat. an, er schmecke

) a. a. 0 . 
etwas, ist sich aber äber die:Qualităt des Geschmackes nicht klar. Auf dem hinteren Zungenabschnitt werden die Stoffe rechts ebenso richtig und schnell wie links erkannt. Die Hautsensibilität im Bereich des 2. Astes vom V stark herabgesetzt (Nadelstiche werden nur als ganz stumpfe Berührungen empfunden, erst bei tiefem Stechen entsteht Schmerz). Die gleiche Herabsetzung in der Nase, ferner in der rechten Mundhöhle am Palatum durum und molle, an der Gingiva des Oberkieferfortsatzes, der Schleimhaut der Backe und auch auf der rechten Zungenhälfte. Sonst ist die Sensibilität an anderen Stellen normal, desgleichen die Bewegung der Zunge, Uvula, Gesichts- und Körpermusculatur. Antiluëtische Cur, die bald Besserung brachte.

Die Diagnose lautete auf einen Tumor am Foramen opticum, der sich vom medialen Rande desselben, wo er den Opticus und Olfactorius comprimirt, an der äusseren Wand des Sinus sphenoidalis dexter herab zum Foramen rotundum erstreckte, wo er auf den N. supramaxillaris einen Druck ausübte. In Rücksicht auf die Anästhesie der rechten Zungenhälfte einen Druck auch auf den N. inframaxillaris am Foramen ovale anzunehmen, hält Salomonsohn für nicht nöthig, da Wolf bei einer Durchtrennung der Chorda Anästhesie der Zunge genau so weit wie die Ageusis reichend erhielt. Da nun dieser Tumor weder auf den VII, noch auf den. IX, noch auf den Plexus tympanicus einen Einfluss ausüben könne, so meint Salomonsohn, es bleibe für die Hemiageusis anterior nur die Erklärung übrig, dass die Geschmacksfasern gleichzeitig mit den anderen Fasern des 2. Astes vom Trigeminus comprimirt wurden. Salomonsohn erklärt seinen Fall also als eine Stütze für die Annahme von Schiff.

Gewiss ist er das auch unbedingt, soweit es sich darum handelt, ob die Geschmacksfasern im $V$ überhaupt verlaufen. Dass er aber auch deren Vorhandensein im 2. Quintusast beweisen soll, ist meiner Meinung nach unbegründet.

Zugegeben dass im Bereich der vorderen zwei Drittel der Zunge auch die sensiblen Fasern aus der Chorda und somit nach Salomonsohn aus dem 2. Ast stammten ${ }^{1}$ ), so hat doch noch Niemand die Sensibilität der einen ganzen Zungenhälfte mit der Chorda in Beziehung gebracht. In der Krankengeschichte aber steht ausdrücklich, dass die Sensibilität auf der rechten

1) Wie schon angedeutet wurde, beweist der oben von mir berichtete Fall, dass, wenn die Chorda überhaupt sensib]e Fasern für die Zunge entbält, diese nur aus dem 3. Ast stammen können. 
Zungenhälfte herabgesetzt war. Dies kann trotz der gegentheiligen Ansicht von Salomonsohn nur durch einen Druck auf den Lingualis erklärt werden. Sollte es sich aber hier nur um eine ungenaue Ausdrucksweise handeln und "rechte Zungenhälfte" so viel zu bedeuten haben als "die vorderen zwei Drittel der rechten Zungenhälfte", so kommt man um eine Erkrankung des 3. Astes vom $V$ doch nicht herum, da auch die Sensibilität der Schleimhaut der Backe als stark herabgesetzt angegeben ist. Letztere aber wird bekanntlich vom N. buccinatorius, einem $\mathrm{Z}$ weige des 3. Astes, versorgt. Dieser war also bestimmt auch mit erkrankt, und damit fallen die Schlüsse Salomonsohn's, soweit sie die Localisation der Geschmacksfasern im 2. Ast betreffen, aus demselben Grunde wie im Heusner'schen Fall in sich zusammen.

Es ist also, wie unsere bisherige Betrachtung zeigt, weder experimentell für das Thier noch klinisch für den Menschen unzweifelhaft und eindeutig nachgewiesen, dass die Geschmacksfasern durch den 2. Trigeminusast passiren. Dagegen ist ein ganz sicherer Fall bekannt, der beweist, dass sie wenigstens beim Menschen im 3. Ast zum gemeinsamen Stamm des $V$ verlaufen. Die Beobachtung rührt von Romberg ${ }^{1}$ ) her und die Section wurde von Henle im Beisein von Johannes Müller und Schwann gemacht. Dieser Fall, der an Klarheit alle anderen in der Literatur verzeichneten bei weitem übertrifft, aber fast nur von Erb genügend hervorgehoben ist, ist folgender:

42jährige Frau. Vor vier Jahren schwerer Sturz, seit droi Jahren heftiger Nieskrampf. Objectiver Befund: im Bereich des 1. und 2. Trigeminusastes normale Sensibilität. Die linke Unterlippe, innen und aussen, sowie die linke Seite des Kinns unempfindlich, desgleichen der innere Theil der linken Obrmuschel und der Gehörgang sowie die Haut der linken Schläfe in der Nähe der Haare. Auch die linke Zungenhälfte anästhetisch, die rechte zeigt normale Sensibilität. Dagegen war die linke Zungenbälfte des. Geschmackes ganz beraubt ${ }^{2}$ ). Die mimischen und die Kaubewegungen normal, deş̧leichen die Bewegungen der Zunge. Keine trophischen Störungen. Tod an Wassersucht. Section: Der 3. linke Trigeminusast an der Stelle, wo er in das Foramen ovale tritt, an seiner äusseren Fläche umgeben von einem

1) Romberg, Nervenkrankbeiten. 2. Aufl. S. 293.

2) Aus einer späteren Bemerkung Romberg's (S. 304) gebt hervor, dass nur der vordere Zungenabschnitt geprüft wurde. 
röthlichen, gefässreichen Gewebe, das theils aus Fasern, theils aus sehr kleinen Bläschen bestand. Bei genauerer Betrachtung erweist es sich als ein Exsudat oder eine Wucherung des Neurilemms, ging gegen die Schädelhöble bin allmäblich in die Substanz der Dura mater, gegen das peripherische Ende des Nerven hin in das normale Neurilemm über. Verdickt und geröthet war das Neurilemm, so weit der Nerv in dem Keilbein verlief, auch noch etwas weiter nach abwärts bis zu der Stelle, wo an der hinteren Fläche des Nerven das normale Gangl. oticum sass. So weit das Neurilemm verändert war, erschien auch der Nerv angeschwollen, gelblich gefärbt und vielleicht etwas härter. Die motorische Wurzel war unverändert und verschmolz mit der grösseren Portion erst unterhalb der kranken Stelle. Der Glossopharyngeus war normal.

Aus dieser Beobachtung, die mir völlig einwandsfrei zu sein scheint, geht mit der Sicherheit eines Experimentes hervor, dass die Geschmacksfasern für die Zunge im 3. Quintusast unmittelbar nach seinem Ursprung aus dem G. Gasseri enthalten waren. Diese Beobachtung muss, wie den meisten Autoren, wohl auch Salomonsohn entgangen sein, denn sonst würde er wohl schwerlich die Behauptung aufstellen, es sei unbestritten, dass auch bei totaler Trigeminuslähmung für den Geschmacksverlust nur der 2. Ast in Frage kommen könne.

Mit dem Romberg'schen Fall stimmt nun der von uns oben beschriebene völlig überein. Wir mussten einen kleinen, eng umschriebenen Heerd am 3. Trigeminusast annehmen dort, wo noch seine sämmtlichen Fasern zasammenliegen. Da hier die bei Romberg unversehrt gebliebene motorische Portion total mitgelähmt war, sass der Heerd entweder an jener Stelle, wo die motorische Portion mit der sensiblen schon verschmolzen ist, demnach etwas tiefer als bei Romberg, oder, was uns nach dem Verlauf als wahrscheinlicher erscheint, die Läsion sass an derselben Stelle wie bei Romberg, also noch vor der Vereinigung beider Portionen und die sensible war stärker betroffen als die motorische. Letzteres ist deswegen wahrscheinlicher, weil, wenn ein gemischter Nervenstamm erkrankt, bekanntlich in der Regel die motorischen Fasern mehr leiden als die sensiblen, während es hier sich umgekehrt verhielt.

Gegen die Beweiskraft des Falles kann, so weit ich sehe, nur eine einzige Einwendung gemacht werden. Es wäre möglich, dass die von mir an der erwähnten Stelle angenommene 
Läsion sich auf den Lingualis nach abwärts erstreckt und die Chorda an der Stelle betroffen hätte, wo sie in den Lingualis vom Facialis her eintritt. Dieser Einwand ist aber hinfällig, wenn man den Verlauf unseres Falles berücksichtigt. Wie die Krankengeschichte zeigt, wurde der Beginn der Rückkehr der Sensibilität für alle Zweige des 3. Astes am nämlichen Tage constatirt. Dies ist doch wohl nur denkbar, wenn man annimmt, dass an einer eng umschriebenen Stelle ein kleiner Krankheitsheerd sitzt. Hätte sich die Krankheit von jener Stelle, wo noch sämmtliche Fasern vereint sind, auf die $Z$ weige des 3. Astes, bes. auf den Lingualis noch ein Ende weit erstreckt, dann wäre wohl eine so gleichmässige Wiederkehr der Function unmöglich gewesen.

Da nun trotz gänzlichen Freibleibens des 2. Astes der Geschmack auf den vorderen zwei Drittel der Zunge genau wie bei Romberg völlig erloschen war, so müssen auch in unserem Fall sämmtliche Geschmacksfasern der Chorda an der Schädelbasis den 3. Ast passirt haben.

Weitere Fälle, aus denen der gleiche Schluss mit gleicher Sicherheit abgeleitet werden könnte, konnten wir in der uns zugängigen Literatur nicht finden. Vielleicht gehört hierher jedoch noch folgender von Stamm beobachteter, bei Romberg ${ }^{1}$ ) vitirter Fall.

Bei einem 50jährigen Mann entstand durch eine Neubildung, die vom Keilbein ausging, eine Anästhesie des rechten Quintus, zuerst im 1. und 2. Ast. Nach einiger Zeit wurde auch der 3. Ast betheiligt. Jedoch blieb der Geschmack erhalten, nur fand auf der linken Zungenhälfte die Geschmaeksempfindung schneller statt. Section: Auf dem rechten Flügel des Keilbeins, grade auf dem Foramen ovale, erhob sich eine feste Geschwulst, mit welcher der V da, wo das G. Gasseri sich in drei Aeste spaltet, so verschmolzen tar, dass es unmöglich war, den Nerven aus der umgebenden Masse vollständig herauszupräpariren. Nur in der Gegend des Abganges des Ram. ophthalmicus und in der Gegend des Abganges des 3. Astes liess sich noch deutlich Nervensubstanz unterscheiden.

Romberg führt den Fall sonderbarer Weise als dagegen sprechend an, dass der Zungenast des V Sinnesnerv des Geschmackes sei. Mir scheint, wenn man daraus überhaupt etwas schliessen will, viel eher noch als Romberg's Schluss, der ganz

1) a. 2. 0. S. 299. 
unbegründet ist, der Schluss zulässig zu sein, dass, wenn die Geschmacksfasern an der Schädelbasis den $V$ passiren, sie im 3. Ast vorhanden sein müssen, da gerade da, wo dieser vom Ganglion Gasseri abzweigt, noch deutlich Nervensubstanz vorhanden war, auf deren bereits erfolgte Alteration man sogar die durch Versuche constatirte Verlangsamung in der Geschmackssempfindung beziehen könnte.

Einen Fall von totaler Lähmung des 1. und 2. Astes vom V wahrscheinlich in Folge von Druck einer syphilitischen Geschwulst auf deren Ursprungsstelle an der Schädelbasis oder auf den vorderen Abschnitt des Gangl. Gasseri berichtet Jaffe ${ }^{1}$ ). Der Geschmack war vollkommen erhalten. Da nach unserer Ansicht in einem erkrankten Nerven zuweilen einzelne Fasergattungen trotz totaler Lähmung anderer functionsfähig bleiben können, so ist daraus natürlich mit Sicherheit nichts zu schliessen. Immerhin spricht der Fall mehr dafür, dass die unversehrten Geschmacksfasern in dem intacten 3. Ast verliefen als gerade in dem erkrankten und total gelähmten 2. Ast.

Werfen wir auf das bisher beigebrachte Material, das freilich wegen der grossen Seltenheit uncomplicirter Fälle bisher nur ein sehr spärliches ist, einen Rückblick, so ergiebt sich, dass für den Menschen bisher einzig und allein mit Sicherheit nur die Annahme zulässig ist, dass die Geschmacksfasern der Chorda durch den 3. Trigeminusast in das G. Gasseri und von hier mit der sensiblen Portion in das Gehirn gelangen. Giebt es nun Fälle, die mit der eben ausgesprochenen Ansicht in Widerspruch stehen? Eine derartige Beobachtung hat $\mathrm{Erb}^{2}$ ) gemacht. Er glaubt aus derselben mit Sicherheit schliessen zu können, dass die Geschmacksfasern nicht im 3. Ast verlaufen. Wir führen zunächst die Beobachtung ausführlich an, die, wenn sie nicht noch eine andere Deutung zuliesse, mit der von uns aufgestellten Annahme in einem unlöslichen Widerspruch stehen würde.

Bei einem 40jährigen Mann wurde constatirt: völlige Oculomotoriuslähmung mit Ptosis und Mydriasis, völlige rechtsseitige Abducenslähmung, rechter Facialis intact, Anästhesie des 3. Astes vom V der rechten Seite (die Mitte der Zunge rechts völlig anästhetisch, auf der Spitze dagegen werden

1) Berl. klin. Wochenschr. 1879. No. 43.

2) Arch. f. klin. Medicin. XV. S. 34 Anm. 
Berübrangen und Nadelstiche rechts noch wahrgenommen, wenn auch in vermindertem Grade), Geschmack rechts völlig erhalten. Lähmung der rechtsseitigen Kaumuskeln. Sensibilität in Gebiet des 1, und 2. Astes ganz normal. Sensibilität und Reflexe vom Gaumen normal. Gehör gut. Heilang durch Jodkali.

Das Krankheitsbild führt Erb nicht auf oinen einzigen Krankheitsheerd zurück, sondern auf verschiedene Heerde in den gelähmten drei Nerven. Die Läsion des 3. Astes vom V sucht er da, wo derselbe unterhalb des Gangl. Gasseri bereits die motorische Portion aufgenommen hat, also im Foramen ovale oder dicht unterhalb desselben, also an der unteren Schädelfä̈che. Diese Annahme würde nach Erb erklären, dass der Geschmack erhalten blieb, weil die Chorda und deren Fortsetzung nicht an der Affection Theil genommen; vielleicht sei auch der Rest von Sensibilität an der rechten Zungenspitze auf das Freisein der Chorda zurückzuführen. Aus dieser Beobachtung sei sicher zu schliessen, dass die Geschmacksfasern der Chorda nicht im 3. Ast verliefen, vielmehr spräche sie für die Annahme von Schiff.

Indem wir uns der von Erb gegebenen Deutang seines Falles völlig anschliessen und demnach den Sitz der Läsion wie er gleichfalls im. For. ovale oder dicht unterhalb desselben an der unteren Schädelfläche annehmen, können wir doch dem daraus gezogenen Schlusse, dass die Geschmacksfasern nicht im 3. Aste verliefen, nicht zustimmen. Der Grund ist ein anatomischer und zwar folgender. An der in Betracht kommenden Stelle an der unteren Schädelfläche, zuweilen schon im Foramen ovale selbst, haben den 3 . Ast des $\mathrm{V}$ schon die Fasern verlassen, die zum Gangl. oticum gehen. Dieses liegt bekanntlich hart am Ausgang des Foramen ovale noch oberhalb jener Stelle, wo der 3. Ast sich fast mit Einem Male in seine Zweige auflöst. Jene Fasern brauchen also durch eine Erkrankung an der erwähnten Stelle nicht mitbetroffen za werden. Nimmt man nun an, und dieser Annahme steht nichts entgegen, dass diese zum G. oticum gelangenden Fasern die Geschmackfasern enthalten, deren weiterer Verlauf dann durch das $G$. oticum, den Petros. superf. minor in das Gangl. geniculi N. facialis und von hier in die Chorda tympani wäre, so ist das Erhaltenbleiben der Geschmacksempfindung 
im Falle von Erb völlig erklärt, erklärt auch der Rest der Sensibilität an der Zungenspitze. Ja, man muss sogar sagen, wenn, wie wir oben glauben bewiesen zu haben, die Geschmacksfasern durch den 3. Ast passiren, so müssen sie bei einem Sitz der Erkrankung an der unteren Schädelfläche wie in Erb's Fall bei genügender Kleinheit des Heerdes intact sein, und es muss demnach auch die Geschmacksempfindung an den vorderen zwei Drittel der Zunge in einem solchen Fall vorhanden bleiben. Es ist also Erb's Beobachtung nicht nur nicht im Widerspruch mit unserer Annahme, sondern sie ist vielmehr eher eine Bestätigung derselben. Auch die von Erb für seine Meinung angeführten Experimente Schiff's und Lussana's, dass Durchschneidung des 3. Astes vom $\mathrm{V}$ oder des $\mathrm{N}$. lingualis an der unteren Schädelfläche den Geschmack nicht alterire, sind völlig in Uebereinstimmung mit unserer Annahme. Da hierbei die vom 3. Ast in das G. oticum gelangenden Fasern wohl immer intact bleiben, so kann man eine Geschmacksstörung dabei gar nicht erwarten.

Umgekehrt folgt aber auch daraus, dass die Geschmacksfasern nur für eine kurze Strecke im 3. Ast verlaufen können und ihn dann wieder verlassen müssen. Da sich andere Bahnen dafür nicht finden, so müssen sie in den Fäden, die zum G. oticum führen, enthalten sein, und von diesem ist der oben angenommene Weg durch den Petros. superf. minor und des G. geniculi weitaus der wahrscheinlichste. Derselbe ist ja, wie die obigen Citate beweisen, auch schon vielfach für den Verlauf eines Theiles der Geschmacksfasern von anderen Autoren in Anspruch genommen worden.

Zum Theil in grellem Widerspruch mit dem Schlusse, zu dem wir im Vorbergehenden gekommen sind, stehen die Ansichten von Gowers ${ }^{1}$ ). Waren eine Anzahl deutscher Forscher der Meinung, dass der Glossopharyngeus der eigentliche Geschmacksnerv sei und dass die Fasern der Chorda vermuthlich von ihm stammten, so sagt grade umgekehrt Gower's: "Ich glaube, es wird sich herausstellen, dass Geschmackseindrücke nur durch die Wurzeln des Quintus zum Gehirn gelangen und dass die Lehre, dass die Wurzeln des Glossopharyngeus irgend etwas mit dem

1) Gowers, Vorlesungen über die Diagnostik der Gehirnkrankheiten, Dentsche Uebersetzung von Mommsen. S, 29 . 
Geschmack zu thun haben, ein sonderbarer physiologischer $\mathrm{My}$ thus ist, verschuldet durch eine zu weit gehende Schlussfolgerung aus gewissen anatomischen Thatsachen und zweifelhaften Thierexperimenten." Die Fasern der Chorda, welche nach ihm sicher der Geschmacksnerv für die vordere Zungenpartie ist, lässt er vom Trigeminus durch den N. Vidianus kommen; dagegen sollen die Geschmacksfasern für den Zungengrund vom Ganglion oticum mittelst des Petrosus superfic. minor und des Plex. tympanicus zum Glossopharyngeus gelangen und sich mit den Fasern desselben im Zungengrund vertheilen.

$\mathrm{Zu}$ seiner Ansicht gelangte Gowers durch einen Fall, in welchem eine isolirte Lähmung des einen Trigeminus bestand, die von einer Erkrankung der Wurzel an der Oberfläche des Pons abhängig war, und bei welchem das Geschmacksvermögen auf derselben Seite geschwunden war und zwar nicht nur auf der vorderen Partie der Zunge, sondern auch an der Zungenwurzel, am weichen Gaumen und den Gaumenbögen. Ausser diesem Fall will Gowers noch zwei ähnliche mit dem gleichen Befund beobachtet haben.

Mit seiner Ansicht setzt Gowers sich in Widerspruch mit allen anderen Autoren, die diesen Gegenstand bearbeiteten. Alle übrigen beobachteten, wie wir sahen, entweder bei Trigeminuslähmung überhaupt keine Geschmacksstörung oder solche doch nur am vorderen Zungenabschnitt. Es ist kaum eine andere Lösung dieses Widerspruches möglich, als dass die Fälle von Gowers complicirter waren als er zugeben will. Zur Section gelangte keiner derselben. Ausführlicher publicirt ist nur der erste. Das Original war mir nicht zugänglich, so dass ich mir keine Ansicht darüber bilden konnte. Indessen erklärt Salomonsohn ${ }^{1}$ ) den Fall für weniger klar und die Diagnose der Localisation für so schwierig, dass er die Section nicht entbehren möchte, bevor der Fall für die Frage der Geschmacksinnervation verwerthet werden könne. Ausserdem sind die beiden oben erwähnten eindeutigen Fälle von Lähmung des Glossopharyngeus an der Schädelbasis bei intactem Quintus mit Verlust des Geschmackes auf dem hinteren Zungenabschnitt mit der Gower'schen Annahme gänzlich unvereinbar. Letztere kann daher zur

) a. a. 0. 
Zeit nur als eine geistreiche, aber ganz unbewiesene, ja den vorliegenden Thatsachen ganz widersprechende Idee bezeichnet werden.

Ebensowenig $\mathrm{zu}$ verwerthen ist trotz der gegentheiligen Ansicht des Autors der jüngst publicirte hierher gehörige Fall von Bruns ${ }^{1}$ ), dessen Geschichte in Kurzem folgende ist.

27jähriger Maurer. Scbädeltrauma am 22. Juni 1888 mit fünftägiger Bewusstlosigkeit, rechtsseitige Facialisläbmung, Trommelfellperforation, Blutung und Ausfluss von Gehirnsubstanz aus dem rechten Ohr. Linkes Auge fast amaurotisch, sich aber bald bessernd, während Mydriasis bleibt und Ptosis hinzutritt. Im Uebrigen wurden zu dieser Zeit sonstige Hirnnervenlähmungen nicht constatirt. Zunächst weiter keine Aenderung. Anfang September links neuroparalytische Ophthalmie.

Status am 9. September (11 Wochen nach der Verletzung): Linksseitige neuroparalytische Ophthalmie, Ptosis und Mydriasis, linksseitige Lähmung des Trochlearis und Abducens, rechts Parese des Abducens, links totale Lähmung des Quintus, rechts ist derselbe intact. Facialis links intact, rechts totale peripherische Lähmung desselben. Hörschärfe beiderseits gleich und recht gut (doch heisst es später im Widerspruch biermit: linkes Trommelfell diffus getrübt, alter Mittelobrkatarrh, schon mehrere Jahre vor dem Unfall Ohrensausen und Schwerbörigkeit!). Von Seiten der Vago-Accessorii und Glossopharyngei keine Störung. Schlucken gut. Empfindlicnlreit des Gaumensegels, des Racbens sowie die Reflexe dieser Gegend intact. Allgemeine cerebrale Störungen fehlen. Notilität und Sensibilität der Extremitäten normal. Der Geschmack war auf der ganzen linken Zungenhälfte vorn und hinten intact, auch aw Gaumen, dagegen an denselben Stellen rechts überall erloschen. Die Diagnose lautete auf Schädelbasisfractur mit Verletzung multipler Gehirnnerven.

Seinem Fall glaubt Bruns deswegen besonderes Gewicht beimessen zu können, weil die pathologisch-anatomische Grundlage der Läsionen eine besonders klare und durchsichtige sei, klarer als in den meisten anderen hierher gehörigen Fällen. Er findet nun, dass derselbe mit keiner der bisherigen Theorien über die Innervation des Geschmackes in Einklang zu bringen und unser $W$ issen von dem Verlauf der Geschmacksnerven daher noch wenig sicher sei. Zar Erklärung denkt er deswegen u. a. auch an individuelle Verschiedenheiten. Gewiss ist in dem Fall von Bruns das Vorhandensein von Geschmacksempfindungen auf dem vorderen linken und rechten hinteren Abschnitt der Zunge

1) Archiv f. Psychiatrie ur Nervenheilkunde. Bd. XX. S. 495. 
bei totaler linksseitiger Trigeminuslähmung und angeblich intactem rechten Glossopharyngeus zunächst sehr auffallend. Ist nun aber der Fall wirklich so klar und durchsichtig, wie Bruns annimmt, dass man daraus sichere Schlüsse ziehen kann? Ich kann das nicht zugestehen. Als uninittelbare Folge des Trauma kann nur die rechtsseitige Facialislähmung, die linksseitige Amaurose und Mydriasis berichtet werden, weil diese Symptome von Anfang an bestanden. Andere Hirnnervenlähmungen wurden von dem behandelnden Arzte damals, wie ausdrücklich angegeben, nicht constatirt. Consequenter Weise müssen daher alle anderen von Bruns 11 Wochen später gefundenen Lähmungserscheinungen als secundäre aufgefasst werden, die sich als Folge von durch das Trauma hervorgerufenen chronisch entzündlichen Prozessen ausbildeten. Oder sollten vorher so auffäliige grobe Befunde, wie die eiper totalen Trigeminus- und Abducenslähmung einfach übersehen worden sein? Das ist doch kaum glaublich.

Wenn Bruns sagt „die totale Anästhesie im linken Quintusgebiet kann wohl nur auf eine schwere Quetschung bezw. Zerrung oder Zerreissung des linken $V$ zurückgeführt werden, die wohl auch schon gleich nach der Verletzung Functionsstörungen gemacht hat", so ist das doch nur eine Vermuthung, aber kein "klarer und durchsichtiger Befund". Können wir somit von der Annahme secundärer chronisch entzündlicher Prozesse nicht absehen, so verliert aber der Fall sofort jede Beweiskraft; denn Bruns giebt selbst zu, dass man sich bei entzündlichen Prozessen das Erhaltenbleiben einzelner Fasergattungen erklären könne, nur will er für seinen Fall von einer derartigen Annahme nichts wissen, weil er alle Lähmungserscheinungen als unmittelbar von dem Trauma abhängig betrachtet. Wir sahen aber, dass das nach der Krankengeschichte durchaus nicht unzweifelhaft und sicher ist.

Wir deuten also den Fall so, dass die Geschmacksstörung auf der hinteren Partie der rechten Zungenhälfte von einer Lähmung des rechten Glossopharyngeus herrührt. Diese erklärt sich durch den Bruch des rechten Felsenbeins, da dadurch eine Schädigung des Nerven im Foramen jugulare leicht hervorgerufen werden konnte. Bruns meint zwar, für eine Verletzung des IX lasse sich kein Befund beibringen. Ich weiss indessen nicht, woran 
man eine Lähmung desselben an der Schädelbasis überhaupt erkennen will, wenn nicht an dem Verlust des Geschmackes. Nach den meisten Autoren ist er an seinem Ursprung ein rein sensibler Nerv, die motorischen Fasern werden ihm durch Anastomosen mit dem Facialis und Vago-Accessorius erst später zugeführt. $\mathrm{Ob}$ er ausser den Geschmacksfasern für Zunge, Gaumen a. s. w. noch sensible Fasern führt, ist mindestens zweifelhaft; in den beiden oben erwähnten Fällen von Lähmung des IX war die Sensibilität wenigstens an der Zunge ganz intact. Es kann sich also nur noch darum handeln, ob vielleicht die Sensibilität einzelner Theile des weichen Gaumens und Rachens von ihm her* rührt. Za jenem gehen indessen noch sensible Fasern vom N. palatinus posterior aus dem G. spheno-palatinum des Trigeminus, zum Rachen sensible Fasern vom Vagus, so dass es zweifelhaft ist, ob eine Lähmung des IX immer erhebliche Anästhesie dieser Theile hervorrufen muss. Das sicherste Symptom einer solchen Lähmung ist daher nach meiner Meinung grade die Geschmacksstörung.

Wir nehmen daher im Bruns'schen Fall auch ohne Bedenken eine Lähmung des Glossopharyngeus an und das einzig Auffallende ist daher nur das Erhaltenbleiben des Geschmackes auf den vorderen zwei Drittel der linken Zungenhälfte. Dies würde aber nach Analogie des oben erwähnten Falles von Erb erklärt werden können.

Mag diese Deutung des Bruns'schen Falles auch Manchem etwas gezwungen erscheinen, so sollte man doch, wie mir scheint, bei einem Fall, der nicht eindeutig ist, lieber an eine etwas ungewöhnliche Deutung denken, als ihn gegen ganz sichere Resultate aus ganz klaren Fällen in's Feld führen.

Wir kornmen also, wenn wir auf das Vorhergehende zurückblicken, zu demselben Resultat über den Verlauf der Geschmacksfasern wie schon früher Erb. Nur müssen wir die von ihm als die wahrscheinlichste hingestellte Annahme, dass die Geschmacksfasern vom Gangl. geniculi zum 2. Ast des Trigeminus gelangen, auf Grund des bis jetzt vorliegenden Materials dahin modificiren, dass sie vielmehr in den 3 . Ast gelangen.

Fragen wir uns nun noch zum Schluss, welches der $W$ eg ist, den diese Fasern auf letzterer Strecke passiren, so kann da- 
für aus anatomischen Gründen in Betracht nur der von so vielen früheren Autoren schon herangezogene Petros. superfic. minor kommen, der bekanntlich das G. oticum mit dem Facialis verbindet. Auf dem Wege zu diesem theilt or sich in zwei Zweige, deren einer direct zum G. geniculi führt, während der andere in die Paukenhöhle herabsteigt und sich mit dem Plexus tympanicus verbindet. $Z u$ diesem führt zugleich von der Decke der Paukenhöhle herab ein Fädchen vom Facialis, der Ram. communicans c. Plex tympanic. (Henle). Es können also die Geschmacksfasern des Petros. minor einmal direct zum Facialis gelangen, zweitens aber auch auf dem Umweg durch den Plex. tympanicus. Es dürften wohl beide Wege benutzt werden. Der letztere erklärt zugleich die Angaben von Urbantschitsch u. A., denen es gelang, vom Plex. tympanicus aus durch Reizung desselben Geschmacksempfindungen auszulösen.

Vielleicht findet so auch der oben erwähnte Fall von Carl seine einfache Erklärung, wenn man annimmt, dass bei ihm gerade der Weg durch den Plex. tympanicus von den Geschmacksfasern hervorragend benutzt wurde. Bei dieser Annahme ist er völlig in Einklang mit der von uns vertretenen Ansicht über den Weg dieser Fasern, und ein Zuräckgreifen auf den Glossopharyngeus ganz unnöthig; denn es kann nun eine Reizung der intacten Chorda eine Geschmacksempfindung bei zerstörtem Plexus tympanicus nicht mehr hervorrufen, wohl aber eine sensible Empfindung vorausgesetzt nur, dass die sensiblen Fasern gerade den directen Weg zwischen G. geniculi und oticum benutzen.

Bei unserer Annahme ist auch die auch von Erb für Ausnahmefälle zugelassene Bahn der Geschmacksfasern vom Eintritt der Chorda ab peripherisch mit dem Facialis, dann durch Verbindungszweige zum Auriculo-temporalis (Stich) leichter verständlich. Sie gelangen auf diesem Wege gleichfalls in den Stamm des dritten Astes, während, wenn sie nun noch in den zweiten Ast gelangen sollten, dies nur auf einem sehr complicirten Wege durch das G. oticum u. s. w. möglich wäre.

Es ergiebt sich ferner analog wie für die verschiedenen $\mathrm{Ab}$ schnitte des Facialis und mit demselben Vorbehalt wie bei diesem ') ein diagnostischer Satz für die Localisation eines Erkran-

1) S. bei Erb in Ziemssen, Handbuch. Bd. XII. 1. Hälfte. S. 485 . 
kungsheerdes am 3. Ast des Trigeminus: Ist bei einer Lähmung desselben die Geschmacksempfindung am vorderen Zungenabschnitt erhalten, so hat die Leitungsunterbrechung wahrscheinlich ihren Sitz unterhalb des Abganges der Fasern zum G. oticum (also vermuthlich ausserhalb der Schädelhöhle), ist dagegen jene erloschen, so ist die Leitungsunterbrechung oberhalb des Abganges dieser zu suchen (also vermuthlich innerhalb der Schädelhöhle oder im Foramen ovale.

Das Resultat der vorstehenden Uutersuchung lässt sich in folgende Sätze zusammenfassen.

1) Die Geschmacksempfindung an den vorderen zwei Drittel der Zunge - zuweilen ist der Abschnitt kleiner - wird durch den Lingualis vermittelt.

2) Die betreffenden Fasern verlassen denselben durch die Chorda tympani, mittelst der sie zusammen mit dem Facialis im Canalis Fallopiae verlaufen bis zum Ganglion geniculi oder in dessen Nähe. Von hier treten sie durch den Petrosus superfic. minor direct oder auf dem Umweg durch den Plexus tympanicus (R. communic. N. facialis) - wahrscheinlich auf beiden $W$ egen - zum G. oticum und von diesem durch dessen Verbindungsfäden in den 3. Ast des Trigeminus, mit dem sie durch das G. Gasseri in das Gehirn eintreten. Von dieser Strecke ist der Weg. vom G. geniculi bis zum G. oticum hypothetisch, die andere Strecke ist durch pathologische Beobachtungen sicher nachgewiesen.

3) Für einen anderen $W e g$, bezw. für den Uebertritt in den 2. Ast des V sind keine sicheren Beweise (beim Menschen) vorhanden. Die Möglichkeit individuell verschiedener Bahnen für die Geschmacksfasern ist zwar vorhanden, doch nöthigt uns bisher keine sichere Beobachtung, eine solche anzunehmen.

4) Das. Vorhandensein oder Fehlen von Geschmacksempfindungen am vorderen Zungenabschnitt ermöglicht zuweilen die Diagnose des Sitzes eines Erkrankungsheerdes am 3. Aste des Trigeminus (innerhalb oder ausserhalb der Schädelhöhle).

5) Für den hinteren Zungenabschnitt ist der Glossopharyngeus Geschmacksnerv. Diese Fasern sind von Anfang an in ihm enthalten und kommen nicht vom Trigeminus (Gowers).

6) Das sicherste Zeichen einer Lähmung des Glossopharyn- 
geus an der Schädelbasis ist die Geschmackslähmung am hinteren Zungenabschnitt.

7) Sensibler Nerv für die ganze Zunge ist der N. lingualis. Zur Zungenspitze gelangen jedoch zuweilen sensible Fasern vom 3. Trigeminusast (alle oder einzelne?) nicht direct durch jenen, sondern zusammen mit den Gesehmacksfasern auch auf dem Umweg durch das Ganglion oticum, den Facialis und die Chorda tympani.

\section{III.}

Ueber einen Fall von Myocarcinom des Uterus.

Von Dr. Victor Liebman, Secuudararzt irn Bürgerspital von Triest.

(Bierzu Taf. III - 1V.)

In jüngster Zeit hat Virchow ${ }^{1}$ ) neuerdings die Aufmerksamkeit der Aerzte auf die Mischgeschwülste gelenkt, indem er hervorhob, wie wichtig es sei, dieselben genau kennen zu lernen, um nicht in prognostische Irrthümer zu verfallen.

Unter allen Mischgeschwülsten scheinen die Myocarcinome die seltensten zu sein. Myocarcinom kann ein Neugebilde nur dann henannt werden, weun es erstens aus neugebildetem Muskelgewebe (quergestreifte oder glatte Muskelfasern) und epithelialen Bildungen besteht; wem zweitens auch die Recidiven und die Metastasen, die sich eventuell bilden sollten, ganz und gar den Charakter der primären Geschwulst haben. Berücksichtigt man die histologische Beschaffenheit der secundären Tumoren nicht, so kann man Gefahr laufen ein carcinomatös degenerirtes M yom, dessen Recidive und Metastasen ächte Carcinome sind, Myocarcinom zu benennen, was jedenfalls irrig wäre. -

Durchforscht man nun die einschlägige Literatur in dieser Richtung, so ist die Auslese eine sehr karge.

1) Virchow, Zur Diagnose und Prognose der Carcinome. Dieses Archiv Bd. 111. S.1. 